\title{
Automatic Border Detection Through a Cardiac Cycle to Analyze Left Ventricular Function
}

\author{
P Negrini, P Tomassini, G Cella, M Magrini, M Ercolani, A Mazzarisi, V Gemignani, A Ciampa, \\ P Marcheschi, P Marraccini
}

CNR Institute of Clinical Physiology Pisa, Italy

\begin{abstract}
The evaluation of the ventricular function has a key role in determining therapy and prognosis in patients with heart disease. The standard analysis is limited to quantification of tele-diastolic and tele-systolic frames. An analysis of wall kinetic in each frame of cardiac cycle might allow further information about contractility and diastolic function. The developed algorithms are aimed to achieve an automatic detection of left ventricular wall kinetic during a cardiac cycle in routine clinical activity.
\end{abstract}

\section{Introduction}

In the era of non invasive evaluation of ventricular function, left ventriculography remains one important step of invasive cardioangiographic study. Morphology, kinetic and quantification of regional and global ventricular contraction are critical information together with coronary anatomy for the therapeutical decision making in patients with coronary heart disease. However, several information is generally missed with the standard analysis of ventricular function. In particular the quantification limited to end-diastolic and end-systolic volume only gives a "broad" evaluation of contractility but no details describing velocity and sequence of ventricular kinetics in systolic and diastolic phase. At present the transaction between analogical to digital image acquisition allow to expand the use of mathematical operators and algorithms from single frame to image sequence. In this paper we describe a method for an automatic analysis and quantifications of the left ventricular function throughout a complete cardiac cycle obtained by left ventriculography acquired in the form of a digital sequence. This procedure is very simple to execute and requires no more time than a standard two frames analysis of ventricular function, but can produce more precise information on global and regional diastolic and systolic ventricular function. The computation is quite complex and initially was developed on a parallel computer to reduce computing-time, however, the improvements of hardware electronics heavily increased the processor performance and allow to use this software also with an entry-level computer of today.

\section{Method}

The pre-processing procedure starts with an imagesubtraction' step: for each image I of the sequence, a subtracted image $\mathrm{S}$ is obtained by computing (for each image pixel)

$$
S=\log (g(I) / g(M))
$$

Being $\mathrm{M}$ a 'mask' image acquired before the injection of the contrast medium and at the same phase of the cardiac cycle and $\mathrm{g}(\mathrm{)})$ is the grey-level of an image. The sequence of the subtracted images $\mathrm{S}$, in an ideal case of no patient motion and fixed acquisition-chain characteristics (fixed X-ray generator and video parameters), should contain only the regions of the ventricle filled by the contrast medium. In a real word, however, more unwanted features are present in the subtracted images. A common detrimental source for $\mathrm{S}$ is the automatic adaptation of the video-chain, which is applied in order to optimise the images brightness. The result of the adaptation of the anode current of the X-ray tube on the images is an overall variation of the brightness, so when the subtraction procedure (1) is applied, a mismatch between the image I and the mask M can be introduced. Our procedure minimizes such a mismatch by performing a correction based on a parameter named " $r$ ". This parameter resulted by the ratio between the maxima of the histograms of the distributions of the grey-levels of S and M :

$$
S=\log (g(I))-(\log (g(M))+\log (r))
$$

The spurious structures generated by the possible patient motion can be minimised by applying an additional filtering algorithm. This algorithm is composed by two steps: 1- a threshold operation, 2- a spatial filtering. The step 1 filters out regions of the image whose grey-levels are higher then a threshold $t$. The threshold $t$ is a heuristic value based on the clinical experience; the step 2 is accomplished with a convolution of the subtracted image with a Gaussian mask.

The final subtracted image is then computed as 


$$
S_{\text {Final }}=\left\{\begin{array}{l}
0 \text { if } g(S * G)>t \\
S * G \text { otherwise }
\end{array}\right.
$$

Being $S * G$ the image obtained convolving $S$ with a Gaussian $G$ of suitable chosen width. The resulting sequence of images is further processed by a clusterization procedure, resulting, for each frame of the sequence, in a binary image $C$ containing only the biggest cluster of $S_{\text {Final }}$. The cluster decomposition of $S_{\text {Final }}$ is obtained by searching the 'isles' of connected pixel having the grey-level below a cluster threshold $t_{c}$. The $t_{c}$ value can be either estimated by an automatic algorithm minimizing a suitable cost function or, when this minimization algorithm fails, by a value selected by the user.

The tracking of the ventricular contours is performed on the clusterized images $C_{i}(i=1 \ldots n)$. Physician is requested to indicate a rough contour on the diastolic frame of the selected sequence (3-6 points are generally enough). Next, a tracking of the ventricular contour $\Gamma_{1}$ of the frame is automatically estimated. This is obtained by adapting the points of the contour to the edge of the cluster on the image $C_{1}$, via a Nearest Edge or a $1 \mathrm{D}$ FOAM (First Order Absolute Moment) algorithm [1,2]. We immediately stress that this procedure is more effective in producing accurate contours than the simple extraction of the cluster contours. This is because the cluster can either be not simply connected or containing spurious structures.

In order to track the ventricular contour on the second frame, the contour $\Gamma_{1}$ is automatically 'propagated' onto $C_{2}$ and then adapted to its cluster edge, thus producing $\Gamma_{2}$. The procedure is then iterated up to the last frame of the sequence. The final result is a sequence of ventricular contours, which are used to estimate the sequence of the volumes of the ventricle by standard formulae (i.e. the Single Plane formula) [3].

\section{Results}

We tested the algorithms in 20 ventriculography recorded during routine clinical activity. The total average time of elaboration of a sequence (selection of a sequence, selection of cycle mask, selection of contrast cycle, computation, results display) takes about 2 minutes, the time of computation takes about 15 seconds. A complete automatic and clinically acceptable analysis of ventricular function was obtained in $50 \%$ of the cases. In the remaining sequences the introduction of a user-defined threshold $t_{c}$ was useful for optimizing the computation. Two patients were unable to maintain the apnea thus motion artifacts interfered with the subtraction phase of the algorithm. The physicians of cath-lab judged correct the automatic analysis of cardiac kinetic in 16 of 20 cases $(80 \%)$. An example of the application of the procedure is shown in figure $1,2,3,4$.

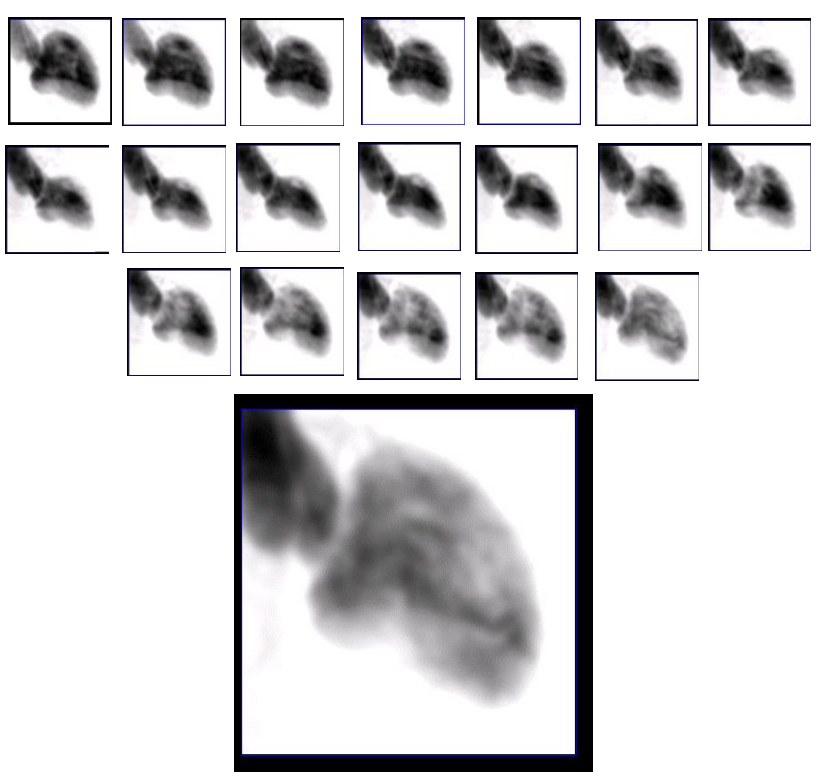

Figure 1. Results of phase triggered image subtraction

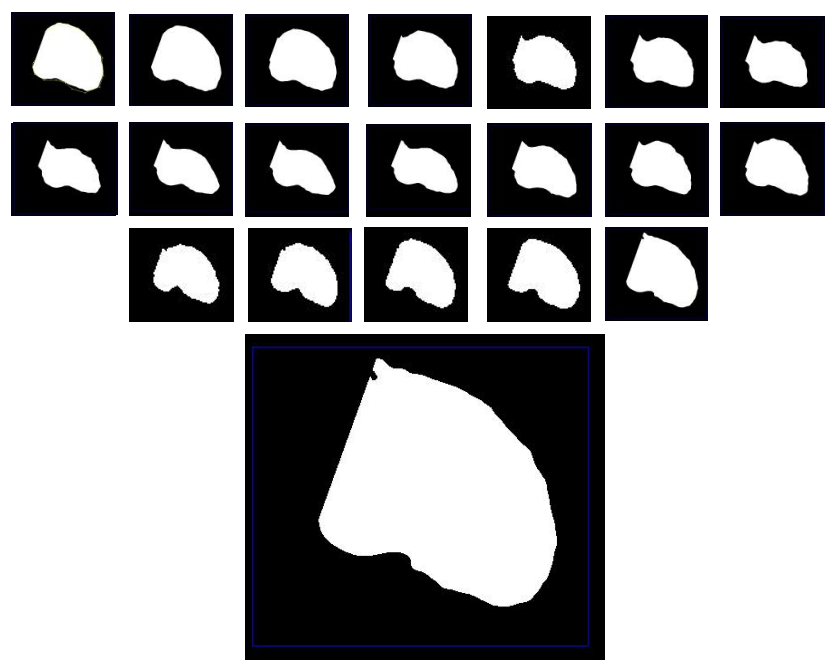

Figure 2. Binarization of image sequence using cluster algorithm. 


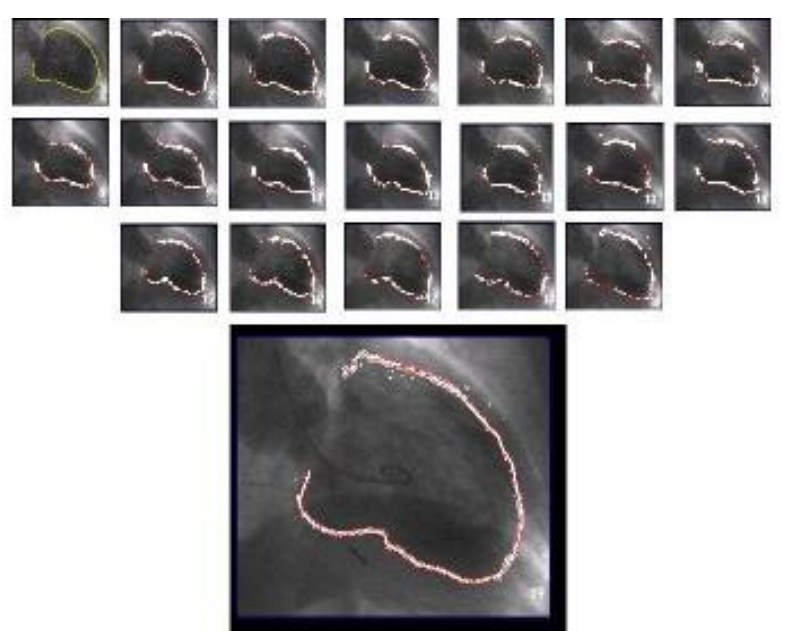

Figure 3: results of automatic ventricular contour tracking
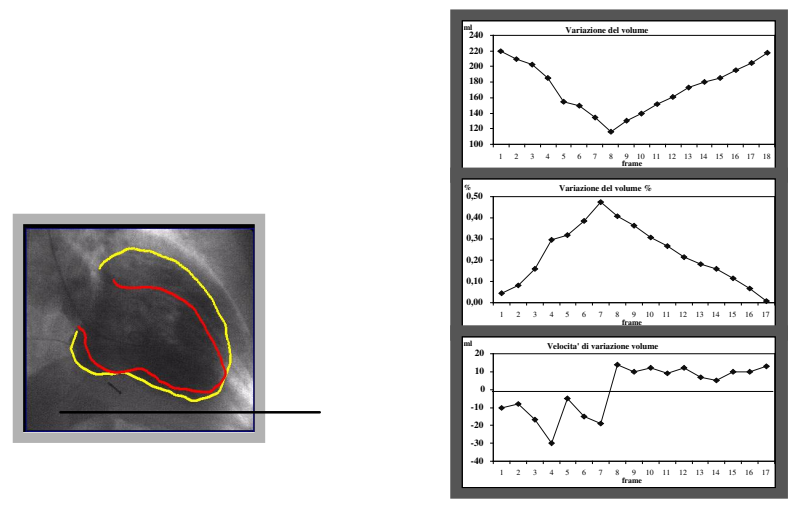

Figure 4: capture screen of the evaluation of global ventricular function presented by the application: telediastolic volume $220 \mathrm{ml}$, systolic volume $116 \mathrm{ml}$, and ejection fraction $47 \%$.

\section{Conclusions}

This experience seems to indicate that algorithm and complex computation on biomedical image sequence can be used even in the routine clinical practice.

Mathematical elaboration of image sequence can improve the level of knowledge and interpretation of medical diagnostic image procedures. In this paper a method for quantitative evaluation of ventricular function through a cardiac cycle is presented. An evaluation frame by frame of global and regional kinetic in systole and diastole could be extracted. This information might be useful for a better understanding of ventricular mechanics and its relation with coronary anatomy.

The accuracy of computed data and its clinical usefulness must be evaluated in further studies.

\section{References}

[1] E. De Micheli, B. Caprile, P. Ottonello and V. Torre, Localization and Noise In Edge Detection, IEEE Trans. Pattern Anal. Machine Intell. 1989;11:1106-11

[2] M. Demi, Contour Tracking by Enhancing Corners and Junctions, Computer Vision and Image Understanding 1996;63:118-16.

[3] Dodge HT, Hay RE, Sandler H: An angiocardiographic method for directly determining left ventricular stroke volume in man Circulation Research 1962;11:739

Address for correspondence.

Paolo Marraccini, MD

CNR Institute of Clinical Physiology

CNR Research Area - Via G. Moruzzi 1

56124 PISA (ITALY)

E-mail address: paolo.marraccini@ifc.cnr.it 
